Research Article

\title{
Nonlinear vibration analysis of axially moving string
}

\author{
Iman Khatami ${ }^{1} \cdot$ Mohsen Zahedi ${ }^{2}$ (i)
}

Received: 29 July 2019 / Accepted: 14 November 2019 / Published online: 25 November 2019

(c) Springer Nature Switzerland AG 2019

\begin{abstract}
In this paper, the nonlinear transverse vibration arising from axially moving string is investigated analytically. Translating string eigenfunctions are employed to reduce a partial-differential equation to a set of second degree of freedom nonlinear systems. The multi-step differential transform method (MsDTM) is proposed in order to find accurate solutions of time-varying length of an axially moving string. To illustrate the applicability and accuracy of MsDTM, the axial motion model is treated with two different sets of parameters. The relationship between transverse displacement, angular velocity and time is obtained and discussed. The effect of the string's speed, damping and tension on the transverse displacement of the string are also taken into consideration.
\end{abstract}

Keywords Nonlinear vibration · Axially moving · Multi-step differential transform method

\section{List of symbols}

A Cross-sectional area

C Constant axial velocity

E Elastic modulus

I Free length between supports

$P$ Initial tension

$t$ Time

$u$ Axial displacement with respect to coordinates translating at velocity $c$

$v$ Transverse displacement with respect to fixed coordinates

$w$ Nondimensional transverse displacement

$x \quad$ Fixed axial coordinate

$\alpha$ Nondimensional initial tension

$\beta \quad$ Nondimensional axial velocity

$\varepsilon \quad$ Strain

$\eta \quad$ Nondimensional time

$\xi$ Nondimensional axial coordinate

$\rho \quad$ Linear density

$\tau \quad$ Nondimensional period

\section{Introduction}

Research into axially continuous material moving at high speed is motivated by various technical applications. Many mechanical devices such as textile fibres, plastic films, power transmission belts, magnetic tapes, elevator cables, paper sheets, band saws, aerial cable tramways, crane hoist cables etc. interfaces with axial movement [1-4]. The main challenge that narrows applications in these devices is limiting the transverse vibration. For example, the quality of the surface of a band saw decreases significantly with vibration of the blade. Another notable case concerns an earthquake in Tohoku in 2011 when elevator ropes oscillated for several minutes due to resonance vibration related to the long period ground motion [5]. Problems connected with the dynamic behaviour of such objects are clear in design and manufacturing and, therefore, understanding the transverse vibrations of axially moving strings is essential.

Despite the diversity of applications in axially moving string, their modelling and analysis has a generalised approach. They study as one-dimensional continua undergoing in-plane motion with no resistance

Mohsen Zahedi, Seyyed.Mohsen.Zahedi@gmail.com | 'Department of Mechanical Engineering, Chabahar Maritime University, Chabahar, Iran. ${ }^{2}$ Department of Computer Engineering, University of Isfahan, Isfahan, Iran. 
to bending. A schematic of the transverse vibration of a taut string stretched with uniform initial tension $P$ between two fixed ends moving with a constant speed $c$ in the longitudinal direction is presented in Fig. 1. Since the gravitational force is sufficiently small compared to the tension force, the equilibrium configuration is a straight line.

The kinetic and potential energies of the string are as follows:

$T=\frac{1}{2} \rho A \int_{0}^{1}\left[v_{T}+c v_{X}^{2}+u_{T}+c^{3}\right] d X$,

$V=\int_{0}^{1} P \varepsilon d X+\frac{1}{2} E A \int_{0}^{l} \varepsilon^{2} d X$

where $u$ and $v$ are the axial and transverse displacements, respectively. The subscript notations represent partial differentiation with respect to the variables $X$ and $T$ as spatial and temporal variables. Integrating the difference for the two energies gives:

$$
\begin{aligned}
\int_{I_{1}}^{I_{2}} L d T= & \int_{I_{1}}^{I_{2}} \int_{0}^{I_{0}} \frac{1}{2} \rho A\left\{v_{T}^{2}+2 c v_{X} v_{T}+c^{3} v_{X}^{2}+u_{T}^{2}+2 c u_{T}+c^{2}\right\} \\
& -P\left\{\left[\left(1+u_{X}\right)^{2}+v_{X}^{2}\right]^{1 / 2}-1\right\} \\
& -\frac{1}{2} E A\left\{\left[1+u_{X}\right]^{2}+v_{X}^{2}+1-2\left[\left(1+u_{X}\right)^{2}+v_{X}^{2}\right]^{1 / 2}\right\} d X d T
\end{aligned}
$$

For $u_{X} \ll 1, v_{X}^{2}<1$, and $v_{X}^{4}<v_{X}^{2}$ the equation of motion is simplified to:

$\rho A v_{\pi T}+2 \rho A c v_{X T}+\left(\rho A c^{2}-P\right) v_{X X}=\frac{3}{2} E\left(v_{X}\right)^{2} v_{X X}(P-A E)=0$

The dimensionless form of Eq. (4) is given by:

$w_{t t}+2 \gamma w_{x t}+\left(\gamma^{2}-1\right) w_{x x}=\frac{3}{2 \kappa^{2}} w_{x}^{2} w_{x x}$

where the dimensionless variables and parameters are:

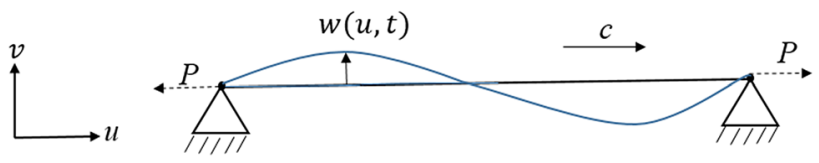

Fig. 1 Schematic of axially moving string

$w=\frac{v}{L}, x=\frac{X}{L}, t=\frac{1}{l} \sqrt{\left(\frac{P}{\rho A}\right)} T, \gamma=\frac{c}{\sqrt{P / \rho A}}, \kappa=\sqrt{\frac{P}{A E}}<1$.

The boundary conditions fixed on both ends are:

$w(0, t)=w(1, t)=0$.

Equation (5), which is a second-order nonlinear partial differential equation, represents an axially moving string with a constant translation speed and a uniform tension. In this model the effect of gravity on the string is ignored, string density does not change with lateral displacement, and the strain remains constant during movement. The closed form solution of $w(x, t)$ is:

$w(x, t)=\sum_{k=1}^{N} q_{k}(t) \sin (k \pi x)$.

By substituting $w(x, t)$ from Eq. (8) into Eq. (5) and integrating at $(0,1)$ intervals, the following set of ordinary differential equations is obtained for temporal coordinates $q_{k}$ :

$$
\begin{aligned}
\ddot{q}_{k} & +8 \gamma \sum_{k+n i s o d d}^{N} \frac{n k}{k^{2}-n^{2}} \dot{q}_{k}(k \pi)^{2} q_{k} \\
& =3 \kappa \sum_{n=1}^{N} \sum_{s=1}^{N} \sum_{m=1}^{N} d_{n s m k} q_{n} q_{s} q_{m}(k=1,2, \ldots, N)
\end{aligned}
$$

where

$d_{n s m k}=\left\{\begin{array}{l}n s m k \pi^{4}, n+s= \pm(m+k), \pm(m-k) \text { orn }-s= \pm(m+k), \pm(m-k) \\ \text { otherwise } \\ 0 .\end{array}\right.$ 
By considering second order truncation in Eq. (9) the following second degree of freedom nonlinear system is found:

$$
\left\{\begin{array}{l}
\ddot{q}_{1}-g \dot{q}_{2}+k_{1} q_{1}+k_{11} q_{1}^{3}+k_{12} q_{1} q_{2}^{2}=0 \\
\ddot{q}_{2}+g \dot{q}_{1}+k_{2} q_{2}+k_{22} q_{2}^{3}+k_{21} q_{1}^{2} q_{2}=0
\end{array}\right.
$$

where

$$
\begin{aligned}
& k_{1}=\pi^{2}\left(1-\gamma^{2}\right), k_{2}=4 \pi^{2}\left(1-\gamma^{2}\right), g=\frac{16 \gamma}{3} \\
& k_{11}=\frac{3}{8} \kappa \pi^{4}, k_{12}=k_{21}=3 \kappa \pi^{4}, k_{22}=6 \kappa \pi^{4} .
\end{aligned}
$$

The axially moving string represents the simplest distributed gyroscopic system. In many cases the geometric and physical nonlinearities are important to take into consideration and linear analysis leads to a serious inaccuracy. The linear theory of transverse vibration is only appropriate for small amplitude motion. Wickert and Mote [6] investigate transverse vibrations of axially moving strings with axial tension. Pakdemirli [7] applies Galerkin's method to discretize the equations of motion and accomplish stability analysis for each approximation by Floquet theory. Ulsoy [8] improves the analytical approximation of transverse vibration for variable speeds by using the multiple scales method. He compares direct perturbation and discretization perturbation in order to calculate the boundaries separating stable and unstable regions. Yurddas et al. [9] obtain stability domains for nonlinear vibrations of axially moving strings with non-ideal mid-support and multi-support conditions. Pellicano [10] detects a wide class of nonlinear phenomena in a power transmission belt system.

Nonlinearity terms in axial string motion make it a mathematically challenging problem to solve. There are several methods of solving nonlinear equations [11-15]. The differential transform method (DTM) is based on a Taylor series expansion and is a useful method for solving linear and nonlinear equations with known initial and boundary condition values [16]. This method has the limitation of convergence. In fact, divergence from the exact solution arises when independent variable values are far from the centre of the Taylor series. To overcome this limitation, the multi-step differential transform method (MsDTM) is applied [17-19]. In this method, the intervals of the independent variables are divided into subintervals. Therefore, the centre of the series changes for each subinterval and independent variable values are no longer far from the centre of the series. This leads to an increase in the accuracy of the results. In fact, one of the main advantages of MsDTM is its ability in providing a continuous representation of the approximate solution.
By applying DTM a series solution is obtained that does not exhibit the real behaviours of the problem but gives a good approximation to the true solution in a very small region. To overcome the shortcoming, in this study an axially moving elastic string with two end supports is analysed by the MsDTM. The effect of two case parameters on the string's transverse displacement is investigated and the results are compared to those obtained by the Runge-Kutta 4th order (RK4) method. The paper is organized as follows: Sect. 2 describes the fundamental formulations of DTM and MsDTM, followed by the implementation of the method to solve the governing equations of axially moving strings based on gyroscopic mode decoupling in Sect. 3. The research ends with final remarks and conclusions.

\section{DTM and MsDTM concepts}

The Taylor series of an analytic function $x(t)$ around point $t_{0}$ is expressed by:

$x(t)=\sum_{k=0}^{\infty} X(k)\left(t-t_{0}\right)^{k}$,

where $X[k]$ is the transformed function of $x(t)$, defined as:

$X(k)=\sum_{k=0}^{\infty} \frac{1}{k !}\left[\frac{d^{k} x(t)}{d t^{k}}\right]_{t=t_{0}}$.

Substituting Eq. (14) into Eq. (13) yields:

$x(t)=\sum_{k=0}^{\infty} \frac{t^{k}}{k !}\left[\frac{d^{k} x(t)}{d t^{k}}\right]_{t=t_{0}}$.

A finite number of summations in Eq. (15) provides a good approximation of $x(t)$. Therefore, Eq. (15) is expressed as:

$x(t)=\sum_{k=0}^{N} \frac{t^{k}}{k !}\left[\frac{d^{k} x(t)}{d t^{k}}\right]_{t=t_{0}}$,

where $N$ represents the finite number of terms providing the approximation of $x(t)$. Table 1 shows some basic transformation functions used in this paper.

In MsDTM, the whole domain is divided into finite subintervals to increase the accuracy, then, for each subinterval, the DTM is applied. The nonlinear differential equation is considered to be:

$g\left(t, x(t), x^{\prime}(t), x^{\prime \prime}(t), x^{\prime \prime \prime}(t), \ldots x^{(p)}(t)\right), \quad t \in\left[t_{f}, t_{l}\right]$, 
Table 1 Differential transform operations

\begin{tabular}{ll}
\hline Original function & Transformed function \\
\hline$a x(t) \pm b y(t)$ & $a X(t) \pm b Y(t)$ \\
$x(t) y(t)$ & $\sum_{l=0}^{k} X(I) Y(k-l)$ \\
$x(t) y(t) z(t)$ & $\sum_{s=0}^{k} \sum_{m=0}^{k-s} X(s) Y(m) Z(k-s-m)$ \\
$\frac{(k+m) !}{k !} X(k+m)$ & $\frac{\omega^{k}}{k !} \cos (\omega(t)+\alpha)$ \\
$\frac{d^{m} x(t)}{d t^{m}}$ & $X[I]=\sum_{I_{n-1}=0}^{I} \sum_{n-2=0}^{I_{n-1}} \cdots \sum_{I_{2}=0}^{I_{3}} \sum_{I_{1}=0}^{I_{2}} G_{1}\left[I_{1}\right] G_{2}\left[I_{2}-I_{1}\right] G_{n-1}\left[I_{n-1}-I_{n-2}\right] G_{n}\left[I-I_{n-1}\right]$ \\
$x_{1}(t) \cdots x_{n-1}(t), x_{n}(t)$ &
\end{tabular}

where $p$ is the highest order of derivation and $t_{f}$ and $t_{L}$ are the first and last points of the interval, respectively. The initial condition is:

$x^{(q)}\left(t_{f}\right)=C_{q}, q=0,1,2, \cdots, p-1$,

where $C_{q}$ is a real number. According to Eqs. (13) and (16), $x(t)$ is revised to:

$x(t)=\sum_{i=0}^{N} X[i]\left(t-t_{0}\right)^{i} \quad t \in\left[t_{f}, t_{l}\right]$.

where $X[i]$ is a transformed function, and $N$ is the number of terms selected by the convergence test. As $x^{(q)}\left(t_{f}\right)=X[q]$, the initial condition is rewritten as:

$X[q]=C_{q}, \quad q=0,1,2, \ldots, p-1$

By dividing the $t$ variable into $T$ subinterval parts, $\left[t_{f}, t_{L}\right]$ is distributed into equal parts $h$, as follows:

$h=\frac{t_{f}-t_{l}}{T}, \quad T=1,2,3, \ldots, p-1$,

Where $T$ is the number of subintervals. By this technique, for each subinterval, a distinct function is defined. These functions are the solutions of the MsDTM.

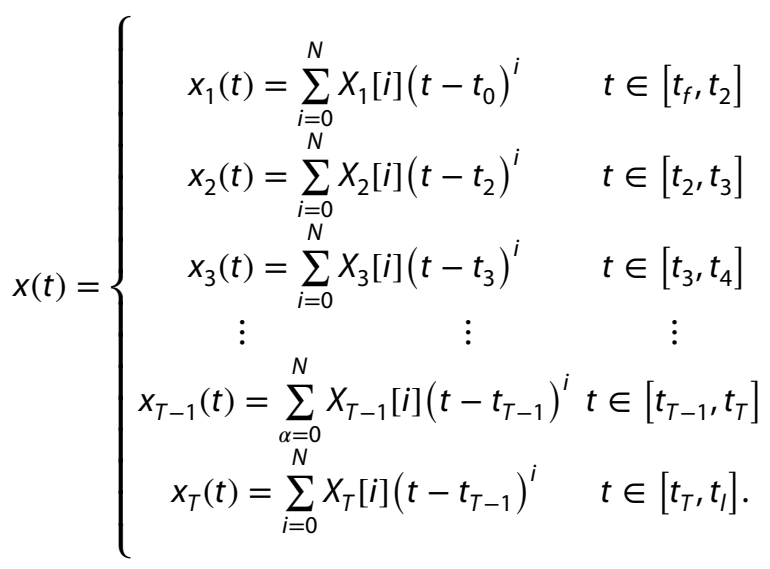

It is clear that the initial condition of $x_{1}(t)$ is $x_{1}^{(q)}\left(t_{f}\right)=X(q)$ and $x_{2}(t)$ is $x_{2}^{(q)}\left(t_{2}\right)=x_{1}^{(q)}\left(t_{2}\right)$. As the value of $x_{1}^{(q)}\left(t_{2}\right)$ is calculated in the first subinterval, $x_{2}^{(q)}\left(t_{2}\right)$ is already known. By continuing the procedure, the initial value of each subinterval is computed.

\section{Axially moving elastic string solution}

The following equations are rewritings of Eq. (11).

$\left\{\begin{array}{l}\ddot{q}_{1}-g \dot{q}_{2}+k_{1} q_{1}+k_{11} q_{1}^{3}+k_{12} q_{1} q_{2}^{2}=0, \\ \ddot{q}_{2}+g \dot{q}_{1}+k_{2} q_{2}+k_{22} q_{2}^{3}+k_{21} q_{1}^{2} q_{2}=0 .\end{array}\right.$

According to Table 1 and Eq. (22), the differential transformation of Eq. (23) for each subinterval is:

$$
\begin{aligned}
\left(Q_{1}\right)_{j}[i+2]= & -\frac{1}{(i+2)(i+1)}\left(-g(i+1) Q_{2, j}[i]+k_{1} Q_{1, j}[i]\right. \\
& +k_{11} \sum_{I_{2}=0}^{i} \sum_{I_{1}=0}^{I_{2}} Q_{1, j}\left[I_{1}\right] Q_{1, j}\left[I_{2}-I_{1}\right] Q_{1, j}\left[i-I_{2}\right] \\
& \left.+k_{12} \sum_{I_{2}=0}^{i} \sum_{I_{1}=0}^{I_{2}} Q_{1, j}\left[I_{1}\right] Q_{2, j}\left[I_{2}-I_{1}\right] Q_{2, j}\left[i-I_{2}\right]\right)
\end{aligned}
$$

$$
\begin{aligned}
\left(Q_{2}\right)_{j}[i+2]= & -\frac{1}{(i+2)(i+1)}\left(+g(i+1) Q_{1, j}[i]+k_{2} Q_{2, j}[i]\right. \\
& +k_{22} \sum_{I_{2}=0}^{i} \sum_{I_{1}=0}^{I_{2}} Q_{2, j}\left[I_{1}\right] Q_{2, j}\left[I_{2}-I_{1}\right] Q_{2, j}\left[i-I_{2}\right] \\
& \left.+k_{21} \sum_{l_{2}=0}^{i} \sum_{l_{1}=0}^{I_{2}} Q_{1, j}\left[I_{1}\right] Q_{1, j}\left[I_{2}-I_{1}\right] Q_{2, j}\left[i-I_{2}\right]\right)
\end{aligned}
$$

where $i=0,1,2, \ldots, N$ and $j=0,1,2, \ldots, T$. The differential transformation of the initial conditions are $\left(Q_{1}\right)_{1}[0]=\left(Q_{2}\right)_{1}[0]=0.1$ and $\left(Q_{1}\right)_{1}[1]=\left(Q_{2}\right)_{1}[1]=0$. The initial condition for each subinterval is defined as: 
$\left(Q_{r}\right)_{j}[0]=q_{1, j-1}(t)$ at $t=t_{j}, j=1,2, \ldots, L$ and $r=1,2$

$\left(Q_{r}\right)_{j}[1]=\frac{d q_{1, j-1}(t)}{d t}$ at $t=t_{j}, j=1,2, \ldots, L$ and $r=1,2$

$t \in\left[t_{f} t_{l}\right]$ is divided into $T$ subintervals of equal size $h$, where $h=\frac{t_{f}-t_{l}}{T}$. According to Eq. (15), by assigning $h=0.1\left(t_{j+1}=t_{j}+h\right),\left(Q_{1}\right)_{j}[\mathrm{i}]$ and $\left(Q_{2}\right)_{j}[\mathrm{i}]$ is calculated where $i=0,1,2, \ldots, N$ and $j=1,2,3, \ldots, T$.

In this research, two sets of parameters are considered. The working parameters of the first case study are $\gamma=0.3$ and $\kappa=0.1$. A non-moving string is selected as a second case study with working parameters $\gamma=0$ and $\kappa=0.1$. Figure 2 shows the vibration amplitude of the first generalized coordinate $\left(q_{1}\right)$ against time obtained by DTM, MsDTM and RK4. The numerical solutions (RK4) are captured for comparison. Figure 2 reveals that the DTM solution has the limitation of convergence for $t>0.5$ compared to MsDTM. The solid blue line represents DTM and the black line with triangular denotes the MsDTM. The approximate

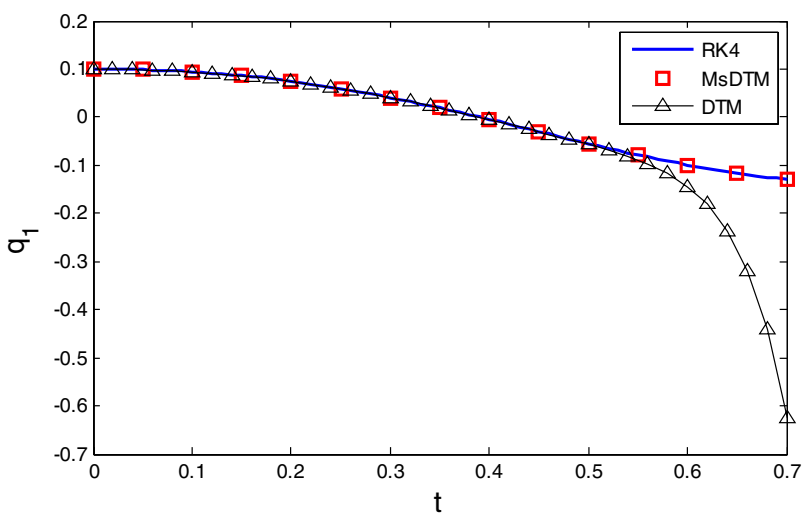

computational time with a Core i7-3770 computer is around $1 \mathrm{~min}$. The number of arithmetic operations increases exponentially with an increasing the number of subintervals and terms.

Since DTM works based on the Taylor series, convergence only happens around the points of the series. This means that by getting away from the points of the series, the results obtained diverge from accurate ones. The DTM is an iterative procedure for obtaining analytic Taylor series solutions of differential equations which taken computationally long time for large orders. In the MsDTM the whole domain is divided into finite subintervals. The Taylor series is applied to each subinterval, which leads to increased precision.

Table 2 shows the values of $\left(Q_{1}\right)_{j}[i]$ and $\left(Q_{2}\right)_{j}[i]$ of the MsDTM for each subinterval of case study 1 that has $\gamma=0.3$ and $\kappa=0.1$.

According to Eq. $(22),\left(q_{1}\right)_{1}(t)$ and $\left(q_{2}\right)_{1}(t)$ for $t \in[00.1]$ are given by:

$$
\begin{aligned}
\left(q_{1}\right)_{1}(t)= & 0.1-0.4642 t^{2}-0.9814 t^{3}+0.5485 t^{4} \\
& +2.44535 t^{5}-0.9545 t^{6}-3.1628 t^{7} \\
& +2.8823 t^{8}+6.1590 t^{9}-8.4593 t^{10} \\
& -18.9226 t^{11}+21.7125 t^{12}+51.5784 t^{13} \\
& -52.4407 t^{14}-121.9363 t^{15} \\
\left(q_{2}\right)_{1}= & 0.1-1.8401 t^{2}-0.2475 t^{3}+6.2377 t^{4}+0.6168 t^{5} \\
- & 10.7241 t^{6}-0.8235 t^{7}+7+22.2011 t^{8} \\
- & 2.6448 t^{9}-67.3669 t^{10}-12.0790 t^{11} \\
+ & 192.4250 t^{12}+39.6011 t^{13}-488.6234 t^{14} \\
- & 106.9306 t^{15} .
\end{aligned}
$$

Fig. 2 Vibration amplitude of the first generalized coordinate

Table 2 The values $\left(Q_{1}\right)_{j}[\mathrm{i}]$ and $\left(Q_{2}\right)_{j}$ i $[$ for each subinterval based on MsDTM for case study 1

\begin{tabular}{llllllll}
\hline$\left(Q_{1}\right)_{j}[\mathrm{i}]$ & $i=0$ & $i=1$ & $i=2$ & $i=3$ & $\ldots$ & $i=15$ & $t \in\left[t_{j} t_{j+1}\right]$ \\
\hline$j=1$ & 0.1 & 0 & -0.464178 & -0.981387 & $\ldots$ & -121.936308 & $t \in[00.1]$ \\
$j=2$ & 0.106348 & -0.118937 & -0.703227 & -0.545598 & $\ldots$ & 109.539458 & $t \in \epsilon[0.10 .2]$ \\
$j=3$ & 0.128988 & -0.269192 & -0.763201 & 0.770163 & $\ldots$ & -11.825739 & $t \in[0.20 .3]$ \\
$\vdots$ & $\vdots$ & $\vdots$ & $\vdots$ & $\vdots$ & $\vdots$ & $\vdots$ \\
$j=L=70$ & 0.888468 & -0.132275 & 0.033530 & -0.762155 & $\ldots$ & -65.418899 & $t \in[9.910]$ \\
\hline$\left(Q_{2}\right)_{j}[\mathrm{i}]$ & $i=0$ & $i=1$ & $i=2$ & $i=3$ & $\ldots$ & $i=15$ & $t \in\left[t_{j} t_{j+1}\right]$ \\
\hline$j=1$ & 0.1 & 0 & -1.840102 & 0.247561 & $\ldots$ & 106.9306622 & $t \in[00.1]$ \\
$j=2$ & 0.116110 & -0.336572 & -1.413069 & 2.480815 & $\ldots$ & 290.948433 & $t \in[0.010 .02]$ \\
$j=3$ & 0.143525 & -0.529961 & -0.463482 & 3.624009 & $\ldots$ & -262.360976 & $t \in[0.020 .03]$ \\
$\vdots$ & $\vdots$ & $\vdots$ & $\vdots$ & $\vdots$ & $\vdots$ & $\vdots$ \\
$j=L=70$ & 1.335080 & -0.175840 & -1.801048 & 1.0869339 & $\ldots$ & 699.129493 & $t \in[9.9910]$ \\
\hline
\end{tabular}




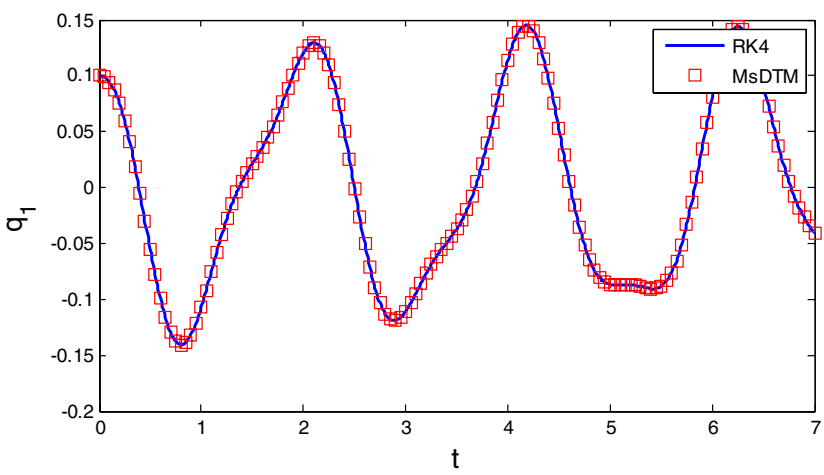

(a)

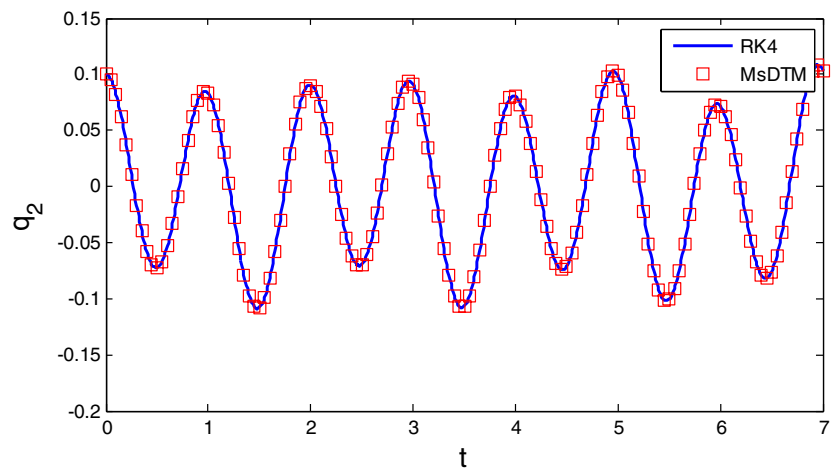

(b)

Fig. 3 Amplitude-time response curve of $\mathbf{a} q_{1}(t)$ and $\mathbf{b} q_{2}(t)$ for case study $1,(\gamma=0.3, \kappa=0.1)$

This procedure is continued for all subintervals to compute $\left(q_{1}\right)_{2}(t),\left(q_{1}\right)_{3}(t), \ldots,\left(q_{1}\right)_{T}(t)$ and $\left(q_{2}\right)_{2}(t)$, $\left(q_{2}\right)_{3}(t), \ldots,\left(q_{2}\right)_{T}(t)$. These terms lead to approximate solutions for $q_{1}(t)$ and $q_{2}(t)$. Figure 3 shows the amplitude-time response curve of $q_{1}(t)$ and $q_{2}(t)$ obtained by MsDTM over long periods of time. The red squares denotes the MsDTM, and the RK4 method is plotted with a blue line to compare the accuracy. It can be seen that the results obtained by the MsDTM are in high agreement with the RK4 method.

Table 3 shows the excellent agreement between MsDTM and RK4 for case study 1 with subintervals $h=0.01$.

Figure 4 shows the amplitude-time response curve of $q_{1}(t)$ and $q_{2}(t)$ for case study $2(\gamma=0, \kappa=0.1)$. The results of the MsDTM have very good agreement with the RK4 method over long periods of time.

The transverse deflection of the axially moving string $w(x, t)$ is derived based on Eq. (8). For $\mathrm{N}=2$ this is equal to:

$w(x, t)=q_{1}(t) \sin (\pi x)+q_{2}(t) \sin (2 \pi x)$.

Figure 5 shows the MsDTM responses for travellingwave speeds $\gamma=0.3, \gamma=1$ and $\gamma=1.2$. The results show that by increasing the axial velocity, the amplitude of the string is increased and moves away from stability.
Table 3 Comparison of the methods for $q_{1}$ and $q_{2}$

\begin{tabular}{lll}
\hline$t$ & $q_{1}$ & $q_{2}$ \\
& $|M s D T M-R K 4|$ & $\mid$ MsDTM $-R K 4 \mid$ \\
\hline 0.00 & 0 & 0 \\
0.10 & $1.11583807701904 \mathrm{e}-05$ & $2.53079750020535 \mathrm{e}-06$ \\
0.20 & $4.08349480843923 \mathrm{e}-05$ & $2.14644866159547 \mathrm{e}-06$ \\
0.30 & $8.48195252197981 \mathrm{e}-05$ & $7.43845939209925 \mathrm{e}-06$ \\
0.40 & 0.000123548871907800 & $1.56649642552020 \mathrm{e}-05$ \\
0.50 & 0.000145756954122098 & $2.76135628617946 \mathrm{e}-05$ \\
0.60 & 0.000150024517666505 & $3.66180750632009 \mathrm{e}-05$ \\
0.70 & 0.000110086646337021 & 0.000123000000005020 \\
0.80 & $1.02560438609844 \mathrm{e}-05$ & 0.000211453750339999 \\
0.90 & 0.000170111030664999 & 0.00224483536052550 \\
1.00 & 0.000371698259129999 & 0.00119862742153400 \\
2.00 & 0.000116123768158991 & 0.000140498934930650 \\
3.00 & 0.000127973890470992 & 0.000188032481573203 \\
4.00 & 0.000828524293291999 & $5.00226342399907 \mathrm{e}-05$ \\
5.00 & 0.00154661854700340 & $4.48169712914948 \mathrm{e}-05$ \\
6.00 & 0.00261909791067511 & 0.000953803536372005 \\
7.00 & 0.00332055424093269 & 0.0935046159621130 \\
8.00 & 0.00455405957375270 & 0.000673000000004490 \\
9.00 & 0.00350554699173430 & 0.00112361743233599 \\
\hline & &
\end{tabular}




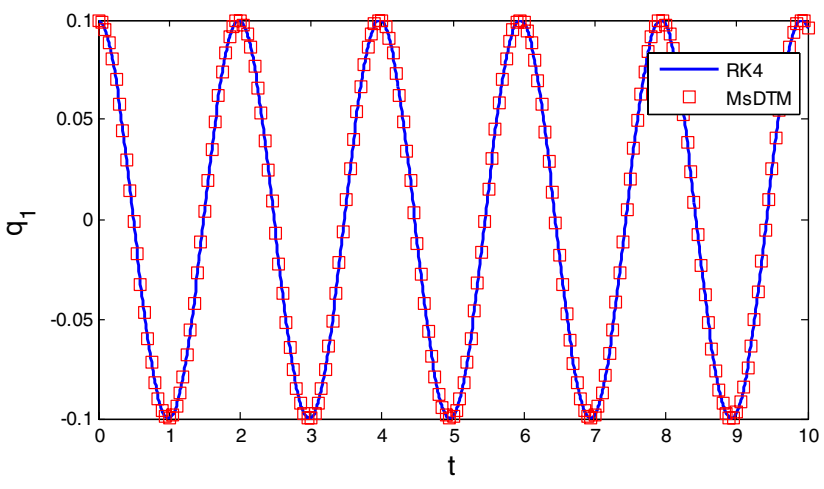

(a)

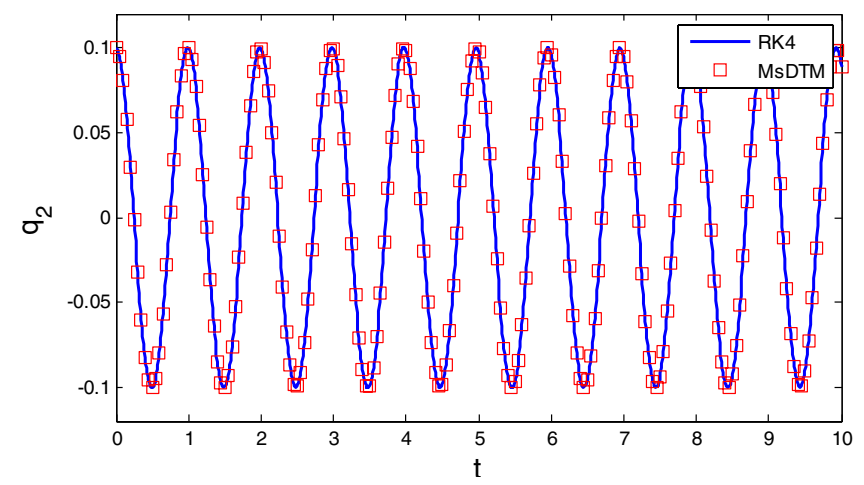

(b)

Fig. 4 Amplitude-time response curve of $\mathbf{a} q_{1}(t)$ and $\mathbf{b} q_{2}(t)$ for case study $2(\gamma=0, \kappa=0.1)$

Fig. 5 MsDTM response of a moving string for travellingwave speeds $\gamma=0.3, \gamma=1$ and $\gamma=1.2$

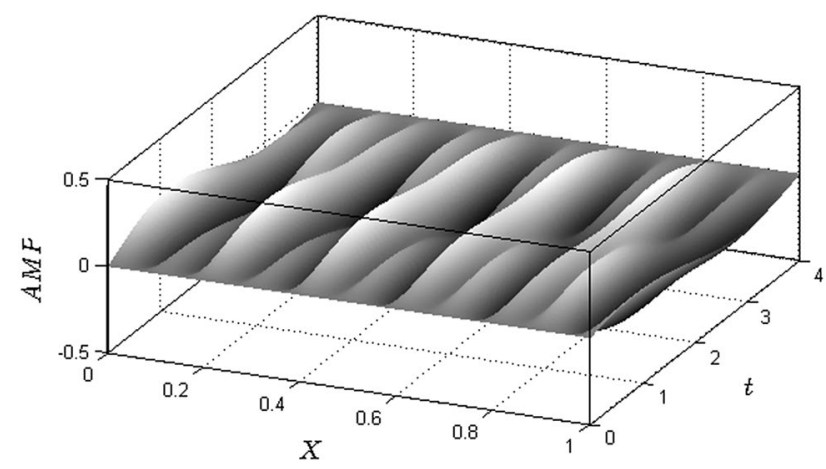

Fig. 6 Transverse deflection of axially moving string $(w(x, t))$ by MsDTM for case $1 \gamma=0, \kappa=0.1$ )

Figure 6 shows the results obtained for the MsDTM for $w(x, t)$, setting $\mathrm{N}=2$.
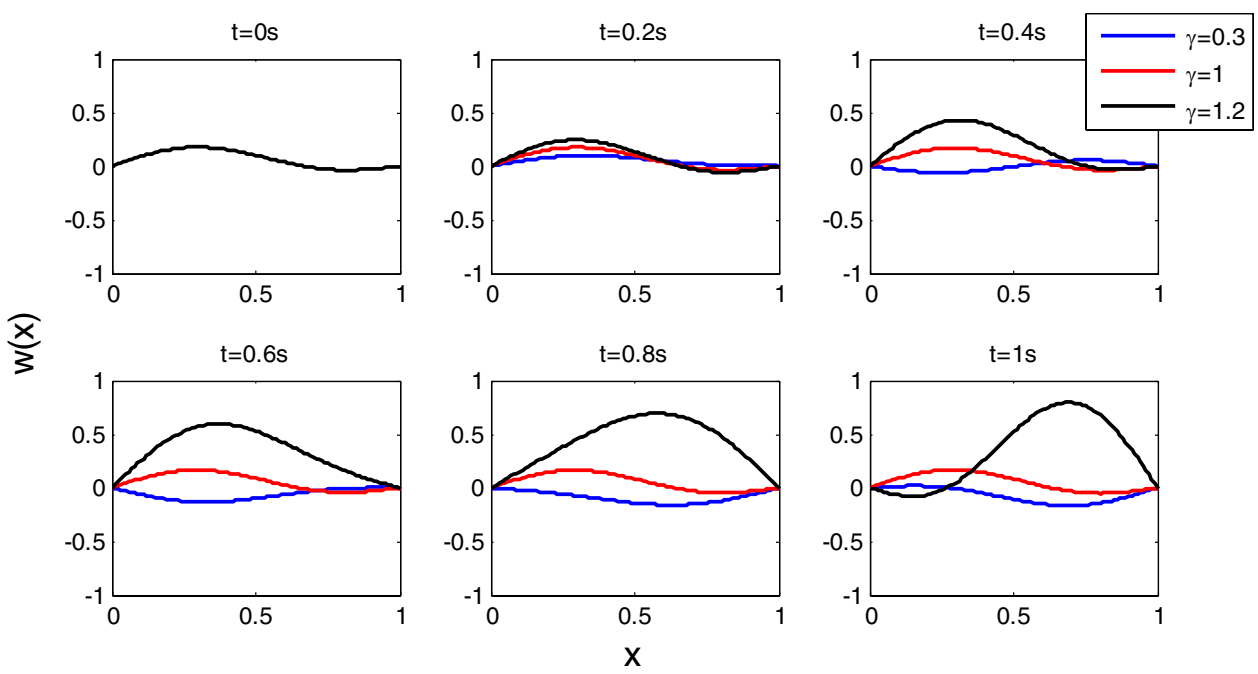

\section{Conclusion}

In this study, the MsDTM method is applied to nonlinear ordinary differential equations for an axially moving string. The two case studies presented in this paper, constant speed and a non-moving string. Both scenarios show excellent comparison with the RK4 numerical approach. It is found that MsDTM improves the convergence of the series solution compare to DTM. The present method can solve differential equations directly with minimum size computation and wide interval of convergence for the series solution. Hence, it is very effective, convenient and accurate for an axially moving string and can be a good alternative idea to treat nonlinear systems. 


\section{Compliance with ethical standards}

Conflict of interest The authors declare that they have no conflict of interest.

\section{References}

1. Marynowski K, Kapitaniak T (2014) Dynamics of axially moving continua. Int J Mech Sci 81:26-41

2. Marynowski K (2008) Non-linear vibrations of the axially moving paper web. J Theor Appl Mech 46:565-580

3. Zhang N, Chen L (2005) Nonlinear dynamical analysis of axially moving viscoelastic strings. Chaos Solitons Fractals 24:1065-1074

4. Ding $H$, Lim CW, Chen L (2018) Nonlinear vibration of a traveling belt with non-homogeneous boundaries. J Sound Vib 424:78-93

5. Miura N, Kohiyama M (2012) Vibration reduction of a buildingelevator system considering the intensity of earthquake excitation. In: World conference on earth quake 2012

6. Wickert JA, Mote CD (2008) Classical vibration analysis of axially moving continua. J Appl Mech 57:738-744

7. Pakdemirli M, Ulsoy AG, Ceranoglu A (1994) Transverse vibration of an axially acceleration string. J Sound Vib 169(2):179-196

8. Yurddas A, Ozkaya E, Boyaci H (2012) Nonlinear vibrations and stability analysis of axially moving strings having nonideal midsupport conditions. J Vib Control 20:518-534

9. Yurddas A, Özkaya E, Boyacı H (2012) Nonlinear vibrations of axially moving multi-supported strings having non-ideal support conditions. Nonlinear Dyn 73:1223-1244
10. Pellicano $\mathrm{F}$ (2005) On the dynamic properties of axially moving system. J Sound Vib 281:593-609

11. Kazemnia M, Zahedi SA, Vaezi M, Tolou N (2008) Assessment of modified variational iteration method in BVPs high-order differential equations. J Appl Sci 8(22):4192-4197

12. Zahedi SA, Fazeli M, Tolou N (2008) Analytical solution of timedependent non-linear partial differential equations using HAM, HPM and VIM. J Appl Sci 8(16):2888-2894

13. Fereidoon A, Zahedi SA, Ganji DD, Rostamiyan Y (2008) Homotopy perturbation method to solving nonlinear WBK equations. Far East J Dyn Syst 10(2):239-254

14. Fazeli M, Zahedi SA, Tolou N (2008) Explicit solution of nonlinear fourth-order parabolic equations via homotopy perturbation method. J Appl Sci 8:2619-2624

15. Saadi F, Azizpour MJ, Zahedi SA (2010) Analytical solutions of kortweg-de vries (KdV) equation. World Acad Sci Eng Technol 69:171-175

16. Malik M, Dang HH (1998) Vibration analysis of continuous system by differential transformation. Appl Math Comput 96(1):17-26

17. Keimanesh $M$, Rashidi MM, Chamkha AJ, Jafari R (2011) Study of a third grade non-Newtonian fluid flow between two parallel plates using the multi-step differential transform method. Comput Math Appl 62(8):2871-2891

18. Khatami I, Zahedi M, Zahedi E (2020) Efficient solution of nonlinear Duffing oscillator. J Appl Comput Mech 6(2):219-234

19. Hatami M, Sheikholeslami M, Domairry G (2014) High accuracy analysis for motion of a spherical particle in plane Couette fluid flow by multi-step differential transformation method. Powder Technol 260:59-67

Publisher's Note Springer Nature remains neutral with regard to jurisdictional claims in published maps and institutional affiliations. 